Research Paper

\title{
Adenovirus-Mediated Wnt5a Expression Inhibits the Telogen-To-Anagen Transition of Hair Follicles in Mice
}

\author{
Yi-Zhan Xing 1, Rui-Min Wang 2, Ke Yang 1, Hai-Ying Guo 1, Fang Deng 1, Yu-Hong Li 1, Ji-Xing Ye 3, Long

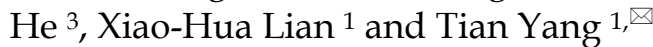 \\ 1. Department of Cell Biology, College of Basic Medical Sciences, Third Military Medical University, Chongqing, China; \\ 2. Experimental Center of Basic Medicine, Chongqing Medical University, Chongqing, China; \\ 3. College of Bioengineering, Chongqing University, Chongqing, China.
}

$\triangle$ Corresponding author: Department of Cell Biology, Third Military Medical University, No. 30, Gaotanyan Street, Shapingba District, Chongqing, China, 400038. Tel.: +86 023687536 14; Fax: +86 023687536 14. Email address: tiany@163.net (Y Tian).

( ) Ivyspring International Publisher. This is an open-access article distributed under the terms of the Creative Commons License (http://creativecommons.org/ licenses/by-nc-nd/3.0/). Reproduction is permitted for personal, noncommercial use, provided that the article is in whole, unmodified, and properly cited.

Received: 2013.02.24; Accepted: 2013.05.08; Published: 2013.05.2I

\begin{abstract}
The canonical $\mathrm{Wnt} / \beta$-catenin pathway plays an important role in hair cycle induction. Wnt5a is a non-canonical Wnt family member that generally antagonizes canonical Wnt signaling in other systems. In hair follicles, Wnt5a and canonical Wnt are both expressed in cells in the telogen stage. Wnt5a has been shown to be critical for controlling hair cell fate. However, the role that Wnt5a plays in the transition from the telogen to anagen stage is unknown. In this study, using whole-mount in situ hybridization, we show that $\mathrm{Wnt} 5 \mathrm{a}$ is produced by several other cell types, excluding dermal papilla cells, throughout the hair cycle. For example, Wnt5a is expressed in bulge and secondary hair germ cells in the telogen stage. Our studies focused on the depilated 8-week-old mouse as a synchronized model of hair growth. Interestingly, overexpression of adenovirus Wnt5a in the dorsal skin of mice led to the elongation of the telogen stage and inhibition of the initiation of the anagen stage. However, following an extended period of time, four pelage hair types grew from hairless skin that was induced by $\mathrm{Wnt} 5 \mathrm{a}$, and the structure of these new hair shafts was normal. Using microarray analysis and quantitative arrays, we showed that the expression of $\beta$-catenin and some target genes of canonical Wnt signaling decreased after Wnt5a treatment. These data demonstrate that $\mathrm{Wnt5}$ a may inhibit the telogen stage to maintain a quiescent state of the hair follicle.
\end{abstract}

Key words: Wnt5a; Hair growth; Wnt signaling pathway; Hair cycle.

\section{Introduction}

The hair follicle (HF) is an important appendage of the skin. HFs renew through the periodic hair cycle $[1,2]$, which consists of the growth (anagen) phase, the regression (catagen) phase, and the rest (telogen) phase. Several signaling pathways are involved in regulating the hair cycle $[3,4]$, and one of the most important of these is the Wnt pathway [5]. Abundant expression of multiple Wnt proteins occurs in the HF and interfollicular epidermis [6], including canonical and non-canonical Wnt proteins.

Inhibitory factors are the dominant regulators in the telogen phase and they maintain HF stem cells in a quiescent state. Once activating signals reach sufficient levels, the HFs are induced to enter the anagen stage $[7,8]$. The canonical Wnt $/ \beta$-catenin pathway plays an important role in the transition from the telogen phase to the anagen phase [9]. Wnt5a, one of typical non-canonical Wnt ligands, begins to be expressed in the dermis at E14.5 during HF development, and subsequently maintained in the dermal papilla (DP) [6]. HF abnormalities, such as inappropriate cytokeratin 10 expression, have been observed in E17.5 embryo skins of Wnt5a-/- mice after skin grafting onto nude mice [10]. These experiments 
suggest that Wnt5a plays a role in controlling HF differentiation. Our previous study has identified the expression of Wnt5a protein throughout the mouse hair cycle. The results show positive staining of Wnt5a in the bulge and the secondary hair germ (HG) during the telogen phase [11], and Wnt5a overexpression inhibits the growth of vibrissa HFs in vitro [11], suggesting that Wnt5a might inhibit the telogen-to-anagen transition in vivo.

In this study, we investigated the role of Wnt5a in the initiation of the anagen phase during the postnatal hair cycle in a depilation-induced synchronization model.

\section{Materials and methods}

\section{I. Animals and adenovirus (Ad) administra- tion}

All animal studies were performed using protocols that were approved by the Third Military University. Female C57BL/6J mice were purchased from the animal facilities of the Third Military Medical University. Ads, including Ad-Wnt5a-GFP and Ad-GFP, were amplified in HEK293 cells and purified by cesium chloride (Amresco, Solon, $\mathrm{OH}, \mathrm{USA}$ ) gradient centrifugation [12]. Ads were injected intradermally into the dorsal skin along the cephalocaudal midline in 8-week-old mice immediately after hair depilation. Each mouse was injected at two sites (dorsocentral and near the tail) with $50 \mu \mathrm{l}$ of Ad solution $\left(10^{8} \mathrm{pfu}\right)$. Additionally, one mouse was injected at four sites (upper, lower, left and right back) with each injection consisting of $25 \mu \mathrm{l}$ of Ad-Wnt5a-GFP solution $\left(10^{8} \mathrm{pfu}\right)$.

\subsection{Whole-mount in situ hybridization}

DIG-labeled probes that detect Wnt5a mRNA were synthesized according to the manufacturer's instructions (DIG RNA Labeling Kit, Roche, Mannheim, Germany). Full-length murine Wnt5a cDNA was subcloned into the pSPT vector, and cRNAs were produced by either NheI linearization and T7 RNA polymerase transcription or NdeI linearization and SP6 RNA polymerase transcription. The back skin tissues from mice at different stages were dissected into strips with a thickness of $1 \mathrm{~mm}$ and fixed in $4 \%$ paraformaldehyde at $4^{\circ} \mathrm{C}$ overnight. After prehybridization for 3.5 hours at $68^{\circ} \mathrm{C}$, the strips were hybridized with probes for 16 hours at $70^{\circ} \mathrm{C}$. Next, the strips were washed, treated with anti-DIG-AP (1:5000; Roche), dehydrated and sealed with glycerin. The images were captured using an E600 microscope (Nikon, Tokyo, Japan). The procedures used for in situ hybridization have been previously described [3].

\subsection{Histology and immunohistochemistry}

All staining assays were performed on $5 \mu \mathrm{m}$ paraffin-embedded sections of back skin. The sections were stained with either hematoxylin and eosin or goat anti-Wnt5a (1:25; AF645, R\&D System, Minneapolis, MN, USA), examined and photographed using a FilC digital camera (Nikon, Tokyo, Japan) that was connected to an E600 microscope (Nikon, Tokyo, Japan).

\subsection{Hair analysis and scanning electron mi- croscopy (SEM)}

Hairs were plucked from normal C57BL/6J mice or from the site of Ad injection 50 days after treatment. Four hair types were placed together and photographed with a Canon digital IXUS 75. For light microscopy analysis, hairs were sealed with resinene and photographed using an E600 microscope (Nikon, Tokyo, Japan) with a FilC digital camera (Nikon, Tokyo, Japan). For SEM, hairs were placed on aluminum stubs, coated with gold platinum using a KYKY SBC-12 Sputter Coater (ZhongKe, Beijing, China) and viewed through an $\mathrm{S}-3400 \mathrm{~V}$ electron microscope (HITACHI, Japan).

\subsection{RNA isolation and microarray analyses}

The following total RNA samples of full-thickness skin tissue were extracted according to standard chloroform/isopropanol methods with the Trizol reagent (Invitrogen, Carlsbad, CA, USA): normal mice at 8 weeks (sample 1); 8 -week-old mice 7 days after hair depilation (sample 2); 8-week-old mice 7 days after hair depilation and intradermal injection of Ad-Wnt5a-GFP (sample 3). Total RNA samples were sent to CapitalBio Co. for mouse genome oligonucleotide microarray analysis (Affymetrix Mouse Genome 4302.0 Array, CapitalBio, Beijing, China). The procedures were performed as described in detail on the website of CapitalBio (http:/ / www.capitalbio. com). The raw array data were normalized and analyzed as previously reported [13].

\subsection{Quantitative real-time polymerase chain reaction ( $Q R T-P C R$ )}

PCR amplification was performed with a SuperGreen PCR kit II (CapitalBio) according to the manufacturer's protocol. The sequences of PCR primers that were used are listed in Supplementary Material: Table S2. The PCR conditions included the following steps: $95^{\circ} \mathrm{C}$ for 5 minute followed by 40 cycles of $95^{\circ} \mathrm{C}$ for 15 seconds, $50-60^{\circ} \mathrm{C}$ for 15 seconds, and $72^{\circ} \mathrm{C}$ for 20 seconds. Differences between measurements were evaluated using the Student's t-test. For all statistical tests, a P-value of $<0.05$ was defined to be statistically significant. 


\section{Results}

\section{I. Wnt5a mRNA expression is dynamic throughout the hair cycle}

To identify the Wnt5a-secreting cells in HFs throughout the hair cycle, we performed whole-mount in situ hybridization on mouse dorsal skin at various stages. At postnatal day 0 (P0), when the $\mathrm{HF}$ is still in the late developmental period, Wnt5a mRNA was detected mainly in the proximal portion of the HF that was migrating into the dermis, with the highest levels detected in the matrix cells. In addition, the basal layer of the epidermis and the middle region of the HF showed moderate Wnt5a mRNA staining (Fig. 1A). The staining in the HF increased by P7, and this increase was most striking in the inner root sheath (IRS) and matrix cells (Fig. 1B). As the HF grew, these cells exhibited the strongest staining for Wnt5a mRNA at P14 (Fig. 1C). By P16, Wnt5a mRNA expression decreased in the regressing follicles, and was restricted to the DP and a few adjacent epithelial cells (Fig. 1D). Wnt5a mRNA staining reached its lowest level during the telogen phase, and weak mRNA staining was detected in DP, HG, and bulge cells at P21 (Fig. 1E). P7 skin showed no detectable Wnt5a transcript using the sense probe (Fig. 1F).

\subsection{Overexpression of $\mathrm{Wnt5a}$ inhibits the ini- tiation of the anagen phase in vivo}

To determine if Wnt5a acts as an inhibitor of HF growth in vivo, the depilated 8-week-old mouse was selected as a synchronized model of hair growth, and the growth of HFs following treatment with an intra- dermal injection of Ad-Wnt5a-GFP or Ad-GFP was compared.

Depilation immediately induces the anagen phase of the hair cycle. Following depilation (7 days or dP7), the control (data not shown) and Ad-GFP-treated group (Fig. 2A, upper row) contained HFs that had entered the mid-anagen phase, as detected by a color change from pink to gray. The back skin of the mice was uniformly black by dP10, and hairs grew out of the skin surface at dP14. However, the skin still remained pink at dP7 in mice belonging to the group that was injected with Ad-Wnt5a-GFP (Fig. 2A, lower row), and the injection boundary in these mice was observed. The entire back skin was black by dP10 with the exception of the treated area, which was still smooth and pink. At dP14, hairs grew from the surface of the surrounding normal skin, but no hair grew from the treated skin. The round, hairless region on these mice slowly and gradually entered the anagen phase beginning at the periphery of the region and moving toward the center in a centripetal manner. The initiation of the anagen phase was accompanied by a change in skin color from pink to black. These results were reproducible over multiple experiments. We examined the expression of GFP in the treated samples at different time points, which means extrinsic Wnt5a. As time went on, its expression level would decreased, which could partly explain the attenuation of inhibition effect. We also injected Ad-Wnt5a-GFP at four different sites in the same mouse and observed the same inhibited phenotype (Fig. 2B). Staining for Wnt5a after Ad-Wnt5a-GFP treatment is shown in Fig. 3A.

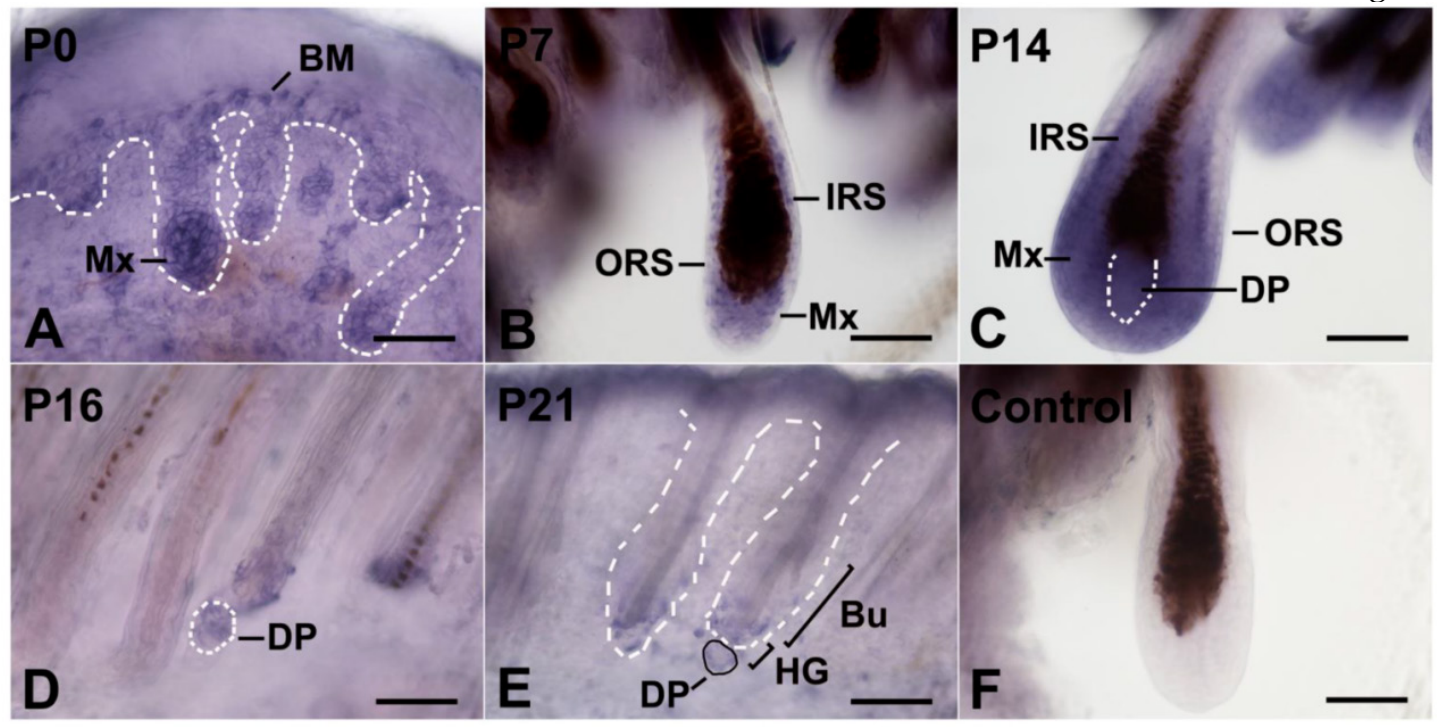

Fig. I. Expression of Wnt5a mRNA in the postnatal hair cycle by whole-mount in situ hybridization. Full-thickness back skins of mice at different stages were dissected into I-mm strips and labeled by DIG-labeled antisense and sense Wnt5a probes. Dotted lines denote the basement membrane that separate the basal layer of the interfollicular epidermis and the HF from the underlying dermis or the DP. (A-E) HFs from skins at P0, P7, PI4, PI6, and P2I. (F) The HF was hybridized with a sense Wnt5a probe. BM, basal membrane; DP, dermal papilla; IRS, inner root sheath; ORS, outer root sheath; Bu, bulge; HG, secondary hair germ; Mx, matrix. Bar $=50 \mu \mathrm{m}$. 


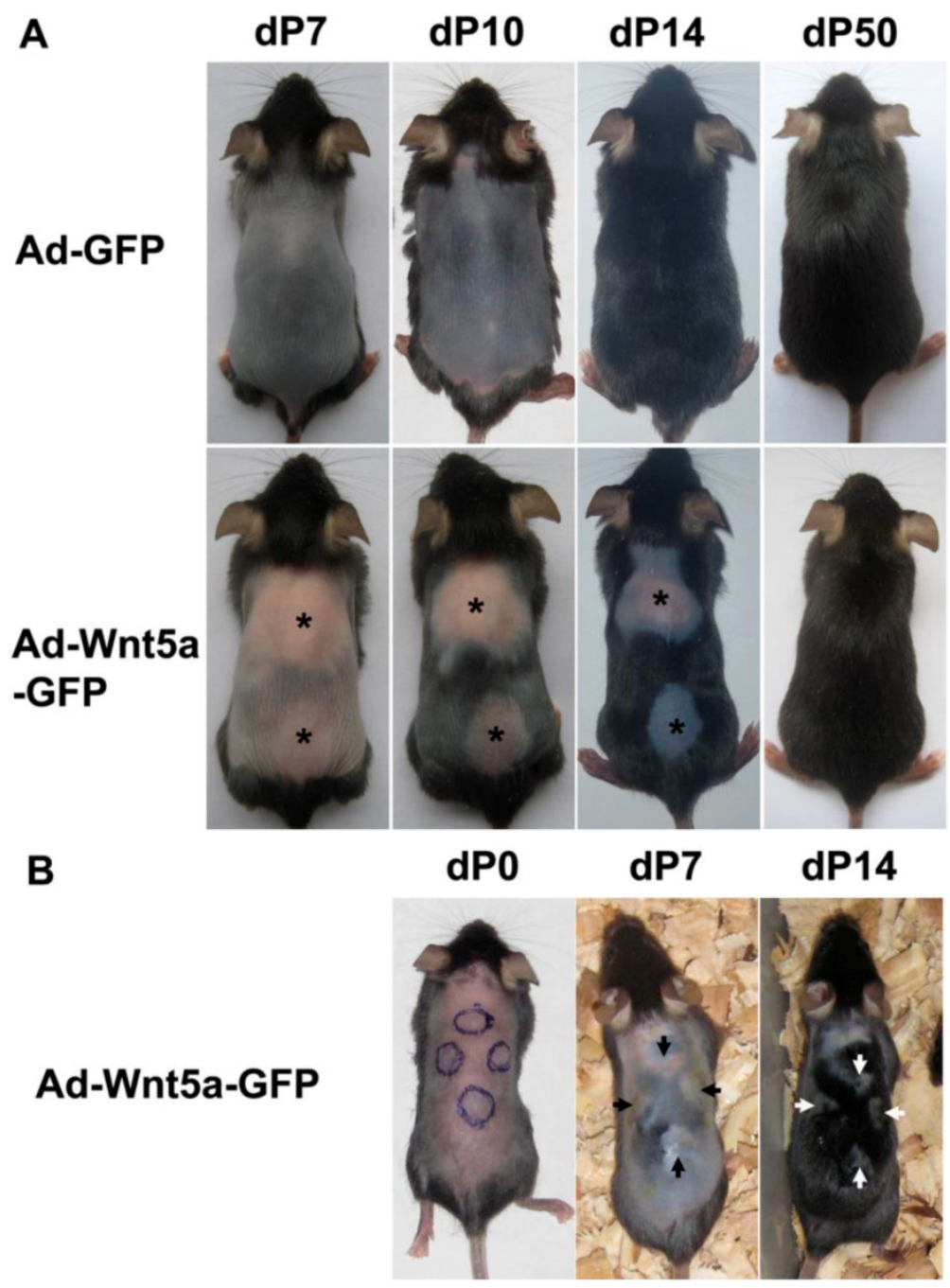

Fig. 2. Inhibition of anagen phase initiation after Wnt5a treatment. Ad-GFP or Ad-Wnt5a-GFP were intradermally injected in the back skin of depilation-induced synchronized C57BL/6] mice. (A) Phenotypes at different time points after Ad-GFP (upper row) or Ad-Wnt5a-GFP (lower row) treatment. An asterisk marks the treated hairless area. (B) Phenotype with four Ad-Wnt5a-GFP-treated sites (arrows) in one mouse.

The morphological structure of the HFs in skin sections was analyzed by light microscopy and used to judge the hair cycle. We investigated dP7 skin sections because these corresponded to the earliest appearance of the inhibited phenotype in vivo. We observed that sections of the Ad-GFP-treated skin (Fig. $3 \mathrm{~B}$, upper) showed characteristics of the mid-anagen phase, including dermis thickening and the extension of HFs deep into the adipose layer of the dermis. However, skin sections from Ad-Wnt5a-GFP-treated mice (Fig. 3B, lower) revealed a clear boundary around the injection region. The HFs in the central area were short and small, and they remained in the telogen phase as judged by analysis of their morphology. However, the HFs in the peripheral region had already entered the mid-anagen phase and formed a multi-layered structure. Together, these data reveal that Wnt5a overexpression prolongs the telogen phase and represses the initiation of the anagen phase in vivo.

\subsection{Overexpression of Wnt5a has no effect on hair type and hair shaft (HS) structure}

After a series of observations, we found that hairs would eventually grow from the hairless regions that had been treated with Ad-Wnt5a-GFP (Fig. 2A), which implied that Wnt5a inhibits the initiation of the anagen phase. To confirm whether Wnt5a influences the formation of the HS structure, we plucked hairs from the back and typed them. The four hair types (zigzag, awl, guard, and auchene) were present in both the Wnt5a group (Fig. 3C) and the control group (data not shown). No significant difference was detected between hairs from the two groups by light microscopy or SEM analysis (Fig. 3D). 


\section{A

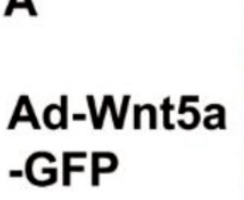

B
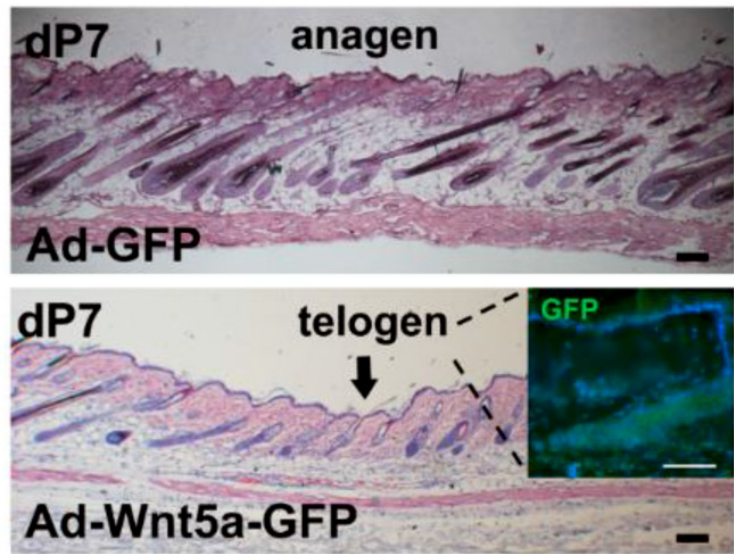

C

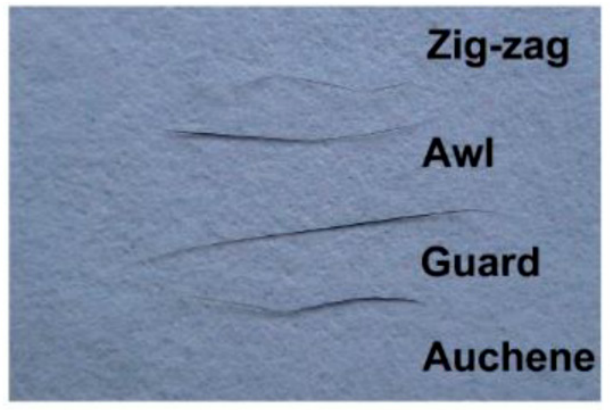

D

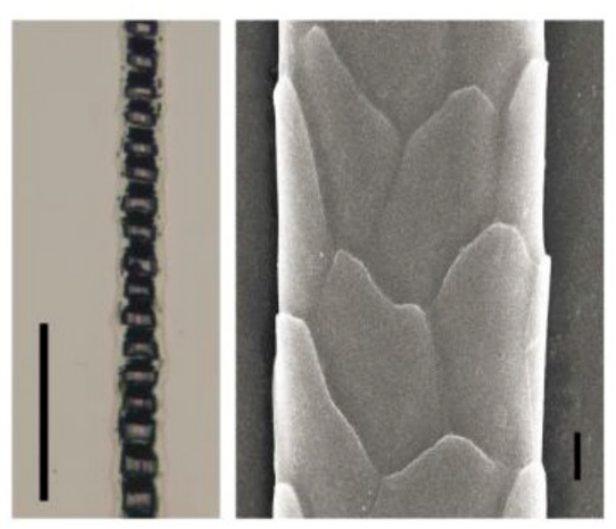

Fig. 3. Morphological comparison in pelage hair after treatment with Ad-Wnt5a-GFP. (A) Wnt5a staining after Ad-Wnt5a-GFP treatment (brown). (B) Hematoxylin and eosin staining of dP7 skin after Ad-GFP or Ad-Wnt5a-GFP treatment. Arrows indicate the central region of injection. GFP staining was shown in the insert. (C) Four pelage hair types were present in the Ad-Wnt5a-GFP group. (D) Hair shaft from the Ad-Wnt5a-GFP group was examined by light microscopy and SEM. Bar $=50 \mu \mathrm{m}$.

\subsection{Gene expression profiling by microarray analysis and quantitative analysis of differen- tially expressed genes}

To further investigate the mechanism underlying the observations described above, we performed genome microarray hybridizations with three purified skin samples. Using microarray analysis (Fig. 4A), we observed that the expression of several genes involved in non-canonical Wnt signaling increased in the Ad-Wnt5a-treated group, such as Rhoa (1.06×), Rac2 (2.66×), and Prkcb (2.21×). In contrast, genes related to the canonical $\mathrm{Wnt} / \beta$-catenin pathway generally decreased, including the target genes, such as Myc (-2.05×), Axin2 (-1.89×) (Supplementary Material: Table S1). Consistent with the light microscopy results, the levels of differentiation markers decreased, including the markers Foxn1 (-1.98×), S100a3 (-2.19×), and Dct (-2.37x) (Supplementary Material: Table S1). To confirm the microarray results, the same three samples were examined by qRT-PCR. Compared to a control mid-anagen skin sample (sample 2), the expression levels of Wnt10b and $\beta$-catenin were decreased in skin that had been treated with Wnt5a (sample 3 with prolonged telogen) (Fig. 4B, 4C), suggesting that the activity of the canonical Wnt $/ \beta$-catenin pathway might be inhibited in these mice. However, Rac2 expression was dramatically elevated following Wnt5a treatment (Fig. 4D), indicating that Wnt5a might initiate Rac2-related signaling.

\section{Discussion}

Canonical Wnt signaling plays an important role in triggering the transition from telogen to anagen during the hair cycle. However, the function of non-canonical Wnt in this process has not been elucidated. In this study, we demonstrated that Wnt5a, a representative non-canonical Wnt protein, prevents the telogen to anagen transition in vivo, which might involve the inhibition of canonical Wnt signaling.

The proliferative matrix and differentiating IRS cells had concentrated Wnt5a mRNA distribution. All of these cells express the Frizzled receptor [14], which serves as receptor in the Wnt signaling pathway. These findings suggest that Wnt5a may mainly act in an autocrine manner in these cells. Additionally, Greco et al. confirmed that the activation of the HG promotes the anagen phase [15]. DP is adjacent to the HG, and the interaction between the epithelium and the mesenchyme is indispensable for the regeneration of HFs. Thus, Wnt5a may participate in the crosstalk between the DP and the HF epithelium. 

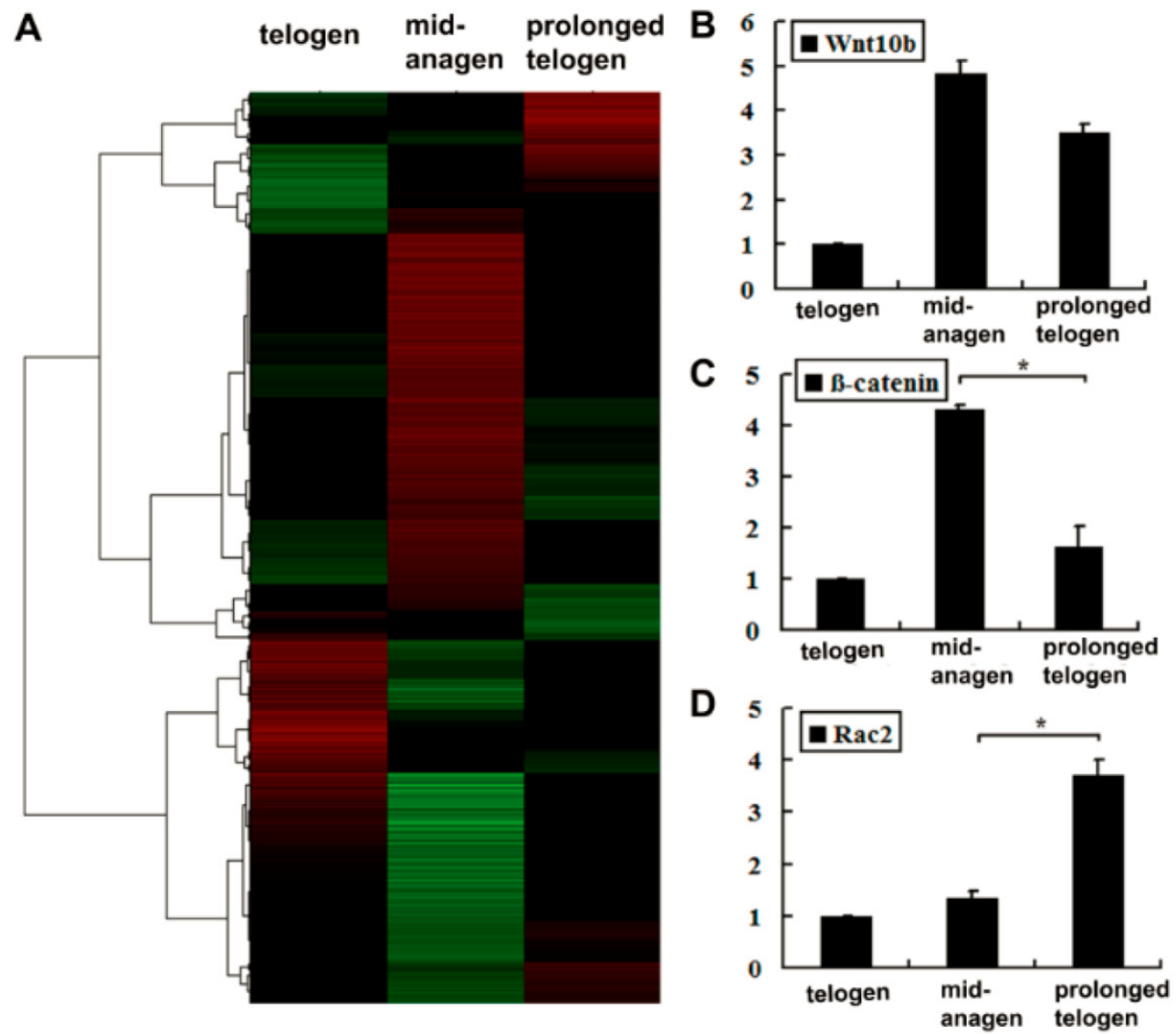

Fig. 4. Microarray and quantitative analysis of several genes related to the Wnt pathway. Full-thickness skin tissues were collected from normal mice at 8 weeks (in telogen); 7 days after hair depilation at 8 weeks (in mid-anagen); 7 days after hair depilation and intradermal injection of Ad-Wnt5a-GFP at 8 weeks (in prolonged telogen). (A) Cluster data of the three samples. (B-D) Three samples were compared by qRT-PCR for Wnt I0b, $\beta$-catenin and Rac2. Values are expressed as the mean $\pm S D(n=3)$. *: P-value $<0.05$.

Previous intradermal injection studies have revealed that some proteins and small molecules can contribute to the transition from the telogen phase to the anagen phase and induce HF growth $[16,17,18]$, but the studies of negative regulators in vivo are rare. Overall, the evidence from previous studies indicates that Wnt signaling usually activates the hair cycle $[7,9,19,20]$. Intriguingly, in this study, the overexpression of Wnt5a markedly interfered with the normal initiation of the anagen phase in vivo. These data suggest that the overexpression of Wnt5a prevents follicle epithelial cells in telogen phase from entering the proliferation or differentiation program and stop the HFs transiently in the telogen phase.

Transient $\beta$-catenin stabilization is essential to maintaining hair growth [21]. Recent reports have suggested that Wnt5a-mediated inhibition of the canonical Wnt pathway, but the mechanism is unclear. Wnt5a-knockout mice show increased $\beta$-catenin signaling in the distal limb [22], that indicates that Wnt5a may inhibit $\beta$-catenin stabilization. Wnt5a can pro- mote $\beta$-catenin degradation through Siah2 and APC in HEK293 cells [23]. Nemeth et al. reported that Wnt5a inhibits canonical Wnt signaling by altering $\beta$-catenin stabilization in hematopoietic stem cells [24]. The mouse whole-genome chip screening demonstrated that genes related to canonical Wnt/ $\beta$-catenin signaling generally decreased after Ad-Wnt5a-GFP injection, including dramatically decreased levels of $\beta$-catenin. These results indicated that $\mathrm{Wnt} / \beta$-catenin signaling is repressed by $\mathrm{Wnt} 5 \mathrm{a}$. Rac, which is a member of the small GTPase superfamily, is involved in the planar cell polarity signaling pathway that is activated by Wnt5a [25]. Although the expression level of the Rac2 gene increased markedly after Wnt5a treatment, the downstream signaling of Wnt5a is different in the context $[26,27,28]$. Therefore, more experiments are required to determine the precise details of Wnt5a signaling.

In conclusion, Wnt signaling plays an important role in inducing the hair cycle. As a non-canonical Wnt signal molecule, Wnt5a is also important in 
mouse hair cycle. Although we found that negative regulation of the hair cycle mediated by Wnt5a occurs via inhibition of canonical Wnt/ $\beta$-catenin signaling, Wnt5a can stimulate several pathways depending on the cell type and phase of the hair cycle. Therefore, we need further studies of the exact mechanism of Wnt5a action in hair growth regulation.

\section{Supplementary Material}

Table S1 - S2.

http://www.medsci.org/v10p0908s1.pdf

\section{Acknowledgments}

We are grateful to Tong-Chuan He (University of Chicago) for kindly providing adenoviruses and helpful discussions. We are also indebted to Cheng-Ming Chuong (University of Southern California) for technical assistance and advice.

\section{Funding}

This work was supported by the National Natural Science Foundation of China [Grant number: 81071309].

\section{Abbreviation}

Ad: adenovirus; DP: dermal papilla; dP: days after depilation; HS: hair shaft; HG: secondary hair germ; HF: hair follicle; IRS: inner root sheath; P: postnatal day.

\section{Competing Interests}

The authors have declared that no competing interest exists.

\section{References}

1. Alonso L, Fuchs E. The hair cycle. J Cell Sci. 2006; 119: 391-3.

2. Muller-Rover S, Handjiski B, van der Veen C, et al. A comprehensive guide for the accurate classification of murine hair follicles in distinct hair cycle stages. J Invest Dermatol. 2001; 117: 3-15.

3. Plikus MV, Mayer JA, de la Cruz D, et al. Cyclic dermal BMP signalling regulates stem cell activation during hair regeneration. Nature. 2008; 451: 340-4.

4. Woo WM, Zhen HH, Oro AE. Shh maintains dermal papilla identity and hair morphogenesis via a Noggin-Shh regulatory loop. Genes Dev. 2012; 26: $1235-46$.

5. Blanpain C, Fuchs E. Epidermal stem cells of the skin. Annu Rev Cell Dev Biol. 2006; 22: 339-73.

6. Reddy S, Andl T, Bagasra A, et al. Characterization of Wnt gene expression in developing and postnatal hair follicles and identification of Wnt5a as a target of Sonic hedgehog in hair follicle morphogenesis. Mech Dev. 2001; 107: 69-82.

7. $\mathrm{Li} \mathrm{YH}$, Zhang $\mathrm{K}$, Yang $\mathrm{K}$, et al. Adenovirus-mediated wnt10b overexpression induces hair follicle regeneration. J Invest Dermatol. 2013; 133: 42-8.

8. Myung PS, Takeo M, Ito M, et al. Epithelial wnt ligand secretion is required for adult hair follicle growth and regeneration. J Invest Dermatol. 2013; 133: 31-41.

9. DasGupta R, Fuchs E. Multiple roles for activated LEF/TCF transcription complexes during hair follicle development and differentiation. Development. 1999; 126: 4557-68.
10. Hu B, Lefort K, Qiu W, et al. Control of hair follicle cell fate by underlying mesenchyme through a CSL-Wnt5a-FoxN1 regulatory axis. Genes Dev. 2010; 24: 1519-32.

11. Xing $Y, X u$ W, Yang $K$, et al. Immunolocalization of Wnt5a during the hair cycle and its role in hair shaft growth in mice. Acta Histochem. 2011; 113: 608-12.

12. Luo J, Deng ZL, Luo $X$, et al. A protocol for rapid generation of recombinant adenoviruses using the AdEasy system. Nat Protoc. 2007; 2: 1236-47.

13. Yang $\mathrm{YH}$, Dudoit $\mathrm{S}$, Luu $\mathrm{P}$, et al. Normalization for cDNA microarray data: a robust composite method addressing single and multiple slide systematic variation. Nucleic Acids Res. 2002; 30: e15.

14. Reddy ST, Andl T, Lu MM, et al. Expression of Frizzled genes in developing and postnatal hair follicles. J Invest Dermatol. 2004; 123: 275-82.

15. Greco V, Chen T, Rendl M, et al. A two-step mechanism for stem cell activation during hair regeneration. Cell Stem Cell. 2009; 4: 155-69.

16. Sato N, Leopold PL, Crystal RG. Effect of adenovirus-mediated expression of Sonic hedgehog gene on hair regrowth in mice with chemotherapy-induced alopecia. J Natl Cancer Inst. 2001; 93: 1858-64.

17. Paladini RD, Saleh J, Qian C, et al. Modulation of hair growth with small molecule agonists of the hedgehog signaling pathway. J Invest Dermatol. 2005; 125: 638-46.

18. Sato N, Leopold PL, Crystal RG. Induction of the hair growth phase in postnatal mice by localized transient expression of Sonic hedgehog. J Clin Invest. 1999; 104: 855-64.

19. Shimizu H, Morgan BA. Wnt signaling through the beta-catenin pathway is sufficient to maintain, but not restore, anagen-phase characteristics of dermal papilla cells. J Invest Dermatol. 2004; 122: 239-45.

20. Beaudoin GM, 3rd, Sisk JM, Coulombe PA, et al. Hairless triggers reactivation of hair growth by promoting Wnt signaling. Proc Natl Acad Sci U S A. 2005; 102: 14653-8.

21. Gat U, DasGupta R, Degenstein L, et al. De Novo hair follicle morphogenesis and hair tumors in mice expressing a truncated beta-catenin in skin. Cell. 1998; 95: 605-14.

22. Yamaguchi TP, Bradley A, McMahon AP, et al. A Wnt5a pathway underlies outgrowth of multiple structures in the vertebrate embryo. Development. 1999; 126: 1211-23.

23. Mikels AJ, Nusse R. Purified Wnt5a protein activates or inhibits beta-catenin-TCF signaling depending on receptor context. PLoS Biol. 2006; 4: e115.

24. Nemeth MJ, Topol L, Anderson SM, et al. Wnt5a inhibits canonical Wnt signaling in hematopoietic stem cells and enhances repopulation. Proc Natl Acad Sci U S A. 2007; 104: 15436-41.

25. Katoh M. WNT/PCP signaling pathway and human cancer (review). Oncol Rep. 2005; 14: 1583-8.

26. Dejmek J, Safholm A, Kamp Nielsen C, et al. Wnt-5a/Ca2+-induced NFAT activity is counteracted by Wnt-5a/Yes-Cdc42-casein kinase 1alpha signaling in human mammary epithelial cells. Mol Cell Biol. 2006; 26: 6024-36.

27. Mikels A, Minami Y, Nusse R. Ror2 receptor requires tyrosine kinase activity to mediate Wnt5A signaling. J Biol Chem. 2009; 284: 30167-76.

28. Witze ES, Litman ES, Argast GM, et al. Wnt5a control of cell polarity and directional movement by polarized redistribution of adhesion receptors. Science. 2008; 320: 365-9. 